\title{
Linear pattern of West Nile virus-associated chorioretinitis is related to retinal nerve fibres organization
}

\begin{abstract}
Purpose To clarify the reason for the linear pattern of West Nile virus (WNV)-associated chorioretinitis.

Methods The study included 12 patients (24 eyes) with WNV-associated chorioretinitis. All the patients underwent a complete ophthalmic evaluation, including dilated fundus examination, fundus photography, fluorescein angiography, and indocyanine green angiography. Characteristics of linear streaks, particularly their relationship to the course of retinal and choroidal vessels, and pattern of retinal nerve fibres, were analysed.

Results All patients had bilateral multifocal chorioretinitis with linear clustering of chorioretinal lesions associated with a variable number of scattered lesions. Linear streaks, variable in number and length, originated from the optic disc or its vicinity in most cases. Their course in all cases appeared to closely follow the course of retinal nerve fibres, rather than that of retinal or choroidal vessels. Conclusions Results of our study show that the linear pattern of WNV-associated chorioretinitis is related to retinal nerve fibres organization, suggesting a contiguous spread of WNV virus from central nerve system via the optic nerve fibres to the outer retina, retinal pigment epithelium, and choroid. Eye (2007) 21, 952-955; doi:10.1038/sj.eye.6702355; published online 21 April 2006
\end{abstract}

Keywords: West Nile virus; chorioretinitis; linear pattern; retinal nerve fibres

\section{Introduction}

Recent studies emphasized multifocal chorioretinitis with typical funduscopic and
M Khairallah, S Ben Yahia, S Attia, S Zaouali, A Ladjimi and R Messaoud fluorescein angiographic appearance of chorioretinal lesions as an early and frequent finding in West Nile virus (WNV) infection. ${ }^{1-7}$ Such a chorioretinitis appears to be a specific marker of the disease, particularly in patients who present with meningoencephalitis. ${ }^{8}$ The distribution of chorioretinal lesions have been described as scattered throughout the fundus or in linear streaks. Linear clustering of chorioretinal lesions was found to be a prominent feature, occurring in more than $80 \%$ of eyes with WNV-associated chorioretinitis. ${ }^{5}$

The pathogenesis of WNV-associated chorioretinitis remains to be elucidated. It has been speculated that haematogenous dissemination of WNV to the choriocapillaris during viraemia may seed the choroid to produce a multifocal chorioretinitis with scattered or linear distribution of chorioretinal lesions. The reason for the pattern of distribution of such lesions was unclear, but it was thought that linear streaks followed the course of choroidal vessels. ${ }^{6}$ The aim of the present study was to attempt to clarify the reason for the linear pattern of WNV-associated chorioretinitis.

\section{Materials and methods}

We retrospectively studied the records of 12 patients (24 eyes) with WNV-associated chorioretinitis. All patients had febrile illness (temperature $\geq 39^{\circ}$ ) along with neurologic syndrome ranging from headache to serious neurologic illness, such as meningitis, encephalitis, or acute focal weakness. Infection with WNV was confirmed in all patients by demonstration of WNVspecific IgM in a serum sample using the IgM 


\section{a}

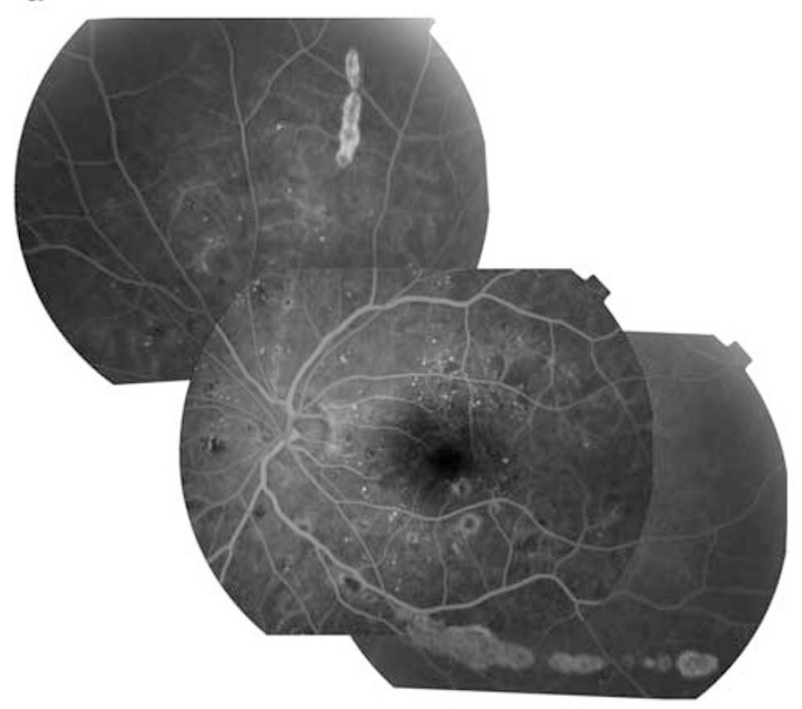

\section{b}

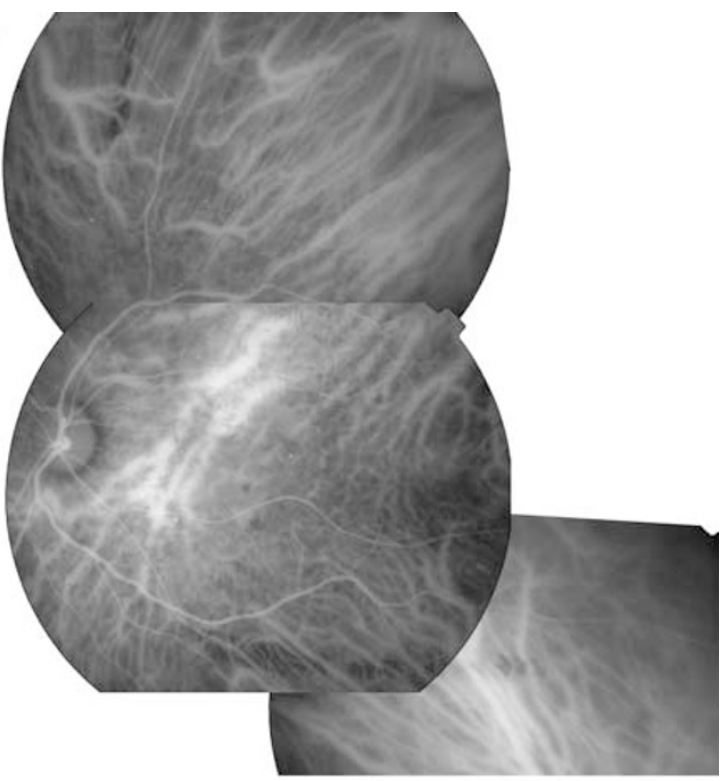

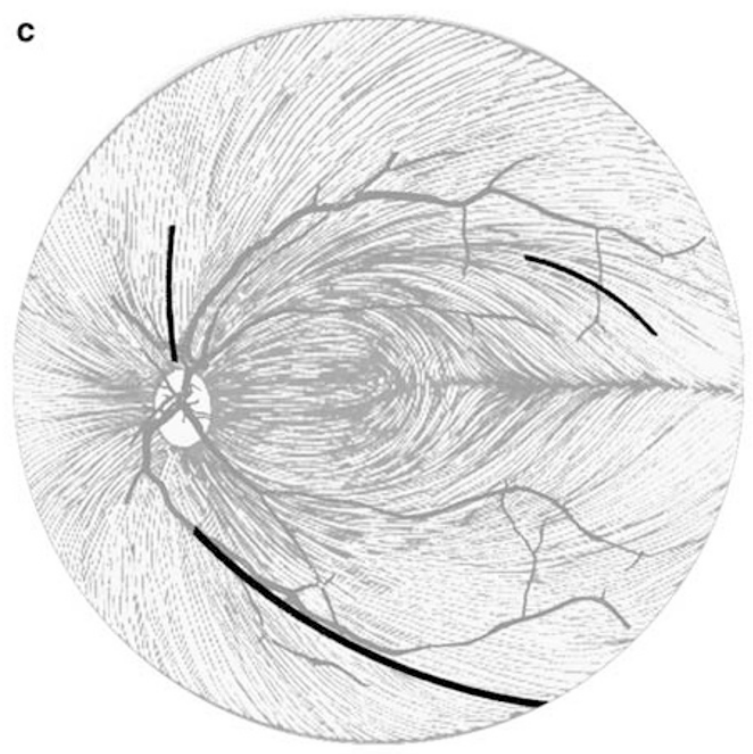

Figure 1 (a) Midphase fluorescein angiogram of the left eye of a diabetic 60-year-old man with a serologically proven WNV infection shows multiple inactive chorioretinal lesions. Note the presence of radial linear streaks superonasally and a curvilinear streak inferoand superotemporally. There is also nonproliferative diabetic retinopathy. (b) Early phase indocyanine green angiogram of the same eye shows hypofluorescent lesions. There is no evidence of orientation of the lesions along the course of choroidal vessels. (c) Schema showing that linear streaks closely follow the course of retinal nerve fibres.

antibody-capture enzyme-linked immunoabsorbent assay (MAC-ELISA).

All these patients underwent a complete ophthalmic evaluation, including dilated fundus examination, fundus photography, fluorescein angiography, and indocyanine green angiography. Linear streaks were analysed in their number, length, origin, location, and course. Fluorescein and indocyanine green angiograms were used to compare the course of linear streaks to that of retinal and choroidal vessels, respectively. A schematic representation of the retinal nerve fibres organization was used to compare the course of linear streaks to that of retinal nerve fibres. All the fundus photographs and angiograms were evaluated independently by two authors (MK, SBY) and in cases of disagreement, consensus was sought with the other authors. Approval by the ethics and research committees of our institution was obtained before initiation of this study. 
lesions associated with a variable number of scattered lesions. The streaks varied in number, from one to more than 3 per eye, and in length approximately from 2 to $15 \mathrm{~mm}$. The streaks originated from the margin or in the vicinity of optic nerve head in most cases. They extended radially in nasal and peripheral fundus or in a curvilinear pattern in posterior temporal fundus. In all cases, the linear arrangement of chorioretinal lesions appeared to closely follow the course of retinal nerve fibres, rather than that of retinal or choroidal vessels (Figures 1 and 2).

\section{Discussion}

The route by which WNV spreads to the retina and choroid is an important issue in the pathogenesis of WNV-associated chorioretinitis. Results of our study clearly show that the linear pattern of WNV-associated chorioretinitis is related to retinal nerve fibres organization. This finding suggests a contiguous spread of WNV from central nerve system via the optic nerve fibres into the eye, rather than a haematogenous dissemination to the choriocapillaris. ${ }^{6}$

In case of neural dissemination, one would expect damage in the nerve fibre layer that could be detected with various tests, including red-free photography, retinal nerve fibre layer analysis at the optic nerve head, optic coherence tomography, or visual field.

Alternatively, the virus might be travelling in neurons and occasionally 'jumping ship' before the axonal tip, leaving the neuron in a spotty fashion, diving directly down to the retinal pigment epithelium and damaging it, leaving the inner retina and neuron intact.

The neural hypothesis for WNV-associated chorioretinitis remains however speculative. Additional studies are warranted to further investigate such a hypothesis, as they may contribute to better understand the pathogenesis of ocular involvement in WNV infection.

\section{References}

1 Bains HS, Jampol LM, Caughron MC, Parnell JR. Vitritis and chorioretinitis in a patient with West Nile virus infection. Arch Ophthalmol 2003; 121: 205-207.

2 Vandenbelt S, Shaikh S, Capone Jr A, Williams GA. Multifocal choroiditis associated with West Nile virus encephalitis. Retina 2003; 23: 97-99.

3 Adelman RA, Membreno JH, Afshari NA, Stoessel KM. West Nile virus chorioretinitis. Retina 2003; 23: 100-101.

4 Hershberger VS, Augsburger JJ, Hutchins RK, Miller SA, Horwitz JA, Bergmann M. Chorioretinal lesions in nonfatal cases of West Nile virus infection. Ophthalmology 2003; 110: 1732-1736.
Figure 2 Red free fundus photograph (a) of the left eye of a diabetic 45-year-old woman with a recent history of WNV infection shows a linear streak (arrows), which follows the course of retinal nerve fibres (b). 
5 Khairallah M, Ben Yahia S, Ladjimi A, Zeghidi H, Ben Romdhane F, Besbes L et al. Chorioretinal involvement in patients with West Nile virus infection. Ophthalmology 2004; 111: 2065-2070.

6 Garg S, Jampol LM. Systemic and intraocular manifestations of West Nile virus infection. Surv Ophthalmol 2005; 50: 3-13.
7 Eidsness RB, Stockl F, Colleaux KM. West Nile chorioretinitis. Can J Ophthalmol 2005; 40: 721-724.

8 Abroug F, Ouanes-besbes L, Letaief M, Ben Romdhane F, Khairallah M, Triki $\mathrm{H}$ et al. A cluster study of predictors of severe West Nile virus infection. Mayo Clin Proc 2006; 81: $12-16$. 